\title{
Correspondencia jerárquica entre las competencias y los resultados de aprendizaje. El caso de "Procedimientos de Construcción”
}

\section{Víctor Yepes}

ICITECH. Equipo de Innovación y Calidad Educativa EXCELCON. Departamento de Ingeniería de la Construcción y Proyectos de Ingeniería Civil. Universitat Politècnica de València, vyepesp@cst.upv.es

\begin{abstract}
This paper aims to establish a hierarchical corresponding structure between the competencies and the learning outcomes of a subject. To this end, after checking the different interpretations that exist between the two concepts, one chooses to consider that the learning outcomes are concretions of the competences for a certain level and that they are the result of the teachinglearning process. In addition, the necessary alignment between the programs of a subject, the acquisition of competencies and learning outcomes and the evaluation of the student, recommends to prioritize the learning results in two levels. As a result of the foregoing, this paper shows the applicability of this hierarchical correspondence to two subjects of the degree of Civil Engineering of the Universitat Politècnica de València: "Construction Procedures I and II".
\end{abstract}

Keywords: competences, learning outcomes, hierarchical correspondence, construction procedures, civil engineering

\footnotetext{
Resumen

El objetivo del artículo es establecer una estructuración de correspondencias jerárquicas entre las competencias y los resultados de aprendizaje de una asignatura. Para ello, tras comprobar las distintas interpretaciones que existen entre ambos conceptos, se opta por considerar que los resultados del aprendizaje son concreciones de las competencias para un determinado nivel y que son el resultado del proceso de enseñanza-aprendizaje. Además, el necesario alineamiento entre los programas de una asignatura, la adquisición de competencias y resultados de aprendizaje y la evaluación del estudiante, aconseja jerarquizar los resultados de aprendizaje en dos niveles. Como resultado de lo anterior, se muestra la aplicabilidad de esta correspondencia jerárquica a dos asignaturas del Grado de Ingeniería Civil de la Universitat Politècnica de València: "Procedimientos de Construcción I y II".
}

Palabras clave: competencias, resultados de aprendizaje, correspondencia jerárquica, procedimientos de construcción, ingeniería civil. 


\section{Introducción}

La Declaración de Bolonia, al tomar como foco el aprendizaje del estudiante, desencadenó un cambio profundo en el sistema universitario. Se trata de proporcionar competencias para que el futuro profesional tenga no solo la capacidad de innovar sino también la de adaptarse a los cambios utilizando y actualizando las competencias requeridas en su vida laboral (Yepes et al., 2012; Torres-Machí et al., 2013). Para asegurar la adquisición de estas competencias, los estudiantes deben alcanzar determinados resultados de aprendizaje. Dichos resultados deben alinearse con los contenidos programáticos, con los sistemas de evaluación y con las mismas competencias. Sin embargo, existe cierta confusión conceptual y organizativa que esta comunicación trata de resolver jerarquizando dichos conceptos y aplicándolo al caso concreto de un par de asignaturas, "Procedimientos de Construcción I y II” del Grado de Ingeniería Civil de la Universitat Politècnica de València (UPV).

Para Lasnier (2000) competencia es “un saber hacer complejo resultado de la integración, movilización y adecuación de capacidades y habilidades (de orden cognitivo, afectivo, psicomotor o social) y de conocimientos, utilizados eficazmente en situaciones que tengan un carácter común”. Según Vila y Poblete (2011) “una competencia supone la integración de una serie de elementos (conocimientos, técnicas, actitudes, procedimientos, valores) que una persona pone en juego en una situación problemática concreta demostrando que es capaz de resolverla". La Universitat Politècnica de València (UPV, 2006) destaca cuatro elementos en la definición de competencia: (a) saber hacer complejo: integración de elementos que permiten realizar tareas complejas; (b) integración: los componentes de las competencias tienen que estar relacionados de una manera sistémica, no lineal ni simplemente yuxtapuestos (puestos uno al lado del otro); (c) adecuación: la competencia activa las capacidades y habilidades pertinentes a la situación y tarea específica; y (d) carácter común de las situaciones: una competencia no es generalizable a todas y cualquiera de las posibles situaciones profesionales.

Los componentes de estas competencias expresan lo que el profesional ejecuta, con qué medios y para qué realiza ese saber hacer. Así, a partir de ellos se seleccionan los contenidos formativos, lo que implica diseñar acciones curriculares que promuevan el conocimiento conceptual, el desarrollo de actitudes y la adquisición de habilidades; acercar al estudiante a los contextos que constituirán su ámbito profesional y social, así como utilizar una metodología que facilite el aprendizaje de prácticas en las que, el estudiante, tenga que movilizar sus recursos. Sobre los componentes que conforman la competencia de un título resulta de interés el trabajo de Pellicer et al. (2013) y Yepes et al. (2016) sobre la adquisición de competencias en un máster en gestión de la construcción.

Así, el perfil académico-profesional de cada titulación debe concretar las competencias transversales y específicas. En ese sentido, el R.D. 1393/1997, de 29 de octubre, por el que se establece la ordenación de las enseñanzas universitarias oficiales, señala en su exposición de motivos que "los planes de estudios conducentes a la obtención de un título deberán tener en el centro de sus objetivos la adquisición de competencias por parte de los

(cc) EY-NC-ND 2018, Universitat Politècnica de València

Congreso IN-RED (2018) 
estudiantes, ampliando, sin excluir, el tradicional enfoque basado en contenidos y horas lectivas". En su Anexo I se indica que, para su correspondiente verificación, las universidades deben presentar "las competencias generales y específicas que los estudiantes deben adquirir durante sus estudios, y que sean exigibles para otorgar el título".

Además, en el Documento Marco sobre la integración del Sistema Educativo Español en el EEES, del Ministerio de Educación, Cultura y Deporte (2003), expone que en un Grado: "Los objetivos formativos de las enseñanzas oficiales de nivel de grado tendrán, con carácter general, una orientación profesional, es decir, deberán proporcionar una formación universitaria en la que se integren armónicamente las competencias genéricas básicas, las competencias transversales relacionadas con la formación integral de las personas y las competencias más específicas que posibiliten una orientación profesional que permita a los titulados una integración en el mercado de trabajo”; y en un Máster: "Los objetivos formativos serán más específicos que los de Grado y deberán estar orientados hacia una mayor profundización intelectual, posibilitando un desarrollo académico disciplinar e interdisciplinar, de especialización científica, de orientación a la investigación o de formación profesional avanzada”.

Con todo, los conceptos de competencia o resultado de aprendizaje no pocas veces se confunden (Yepes, 2017), tal y como se argumentará posteriormente. Para ello la comunicación se centra en su aplicación en las asignaturas "Procedimientos de Construcción I y II", del Grado de Ingeniería Civil impartido en la Escuela Técnica Superior de Ingenieros de Caminos, Canales y Puertos de Valencia (ETSICCP). La comunicación aclara la organización y jerarquía de estas competencias y resultados de aprendizaje en dichas asignaturas.

\section{Objetivos}

Esta comunicación tiene como objetivos los que se relacionan a continuación:

1. Justificar la necesidad de establecer una correspondencia jerarquíca entre las competencias de un título universitario y los resultados de aprendizaje de primer y segundo nivel necesarios para alcanzarlos

2. Mostrar un caso concreto de correspondencia jerárquica aplicado a las asignaturas "Procedimientos de Construcción I y II" del Grado de Ingeniería Civil de la UPV.

\section{Desarrollo de la innovación}

Este apartado plantea como novedad una correspondencia jerárquica entre las competencias y los resultados de aprendizaje, aclarando las diferencias entre estos conceptos. Las competencias específicas son las propias de un título y se orientan a la consecución de un perfil concreto del egresado, que incluyen las competencias básicas o generales y las competencias específicas propiamente. En el caso de los títulos que habilitan para el ejercicio de una actividad profesional regulada, las órdenes ministeriales correspondientes 
hacen referencia a las competencias que se deben requerir. Eso ocurre con el diseño del título de Grado en Ingeniería Civil en la UPV, basadas en lo recogido en el "Anexo I" de la Orden CIN/307/2009 incluye las competencias de carácter general y las competencias de las especializaciones que habilitan para la profesión de Ingeniero Técnico de Obras Públicas, complementándose con otras competencias adicionales dando lugar al listado completo recogido en la actual Memoria de Verificación del título, fechada el 30 de julio de 2015.

Por otra parte, un resultado de aprendizaje sería "una declaración de lo que el estudiante se espera que conozca, comprenda y sea capaz de hacer al finalizar un periodo de aprendizaje". Este concepto se considera como uno de los pilares del proceso de Bolonia (R.D. 1027/2011; ANECA, 2014). Sin embargo, esta noción, muy relacionada con el estudiante, se confunde en ocasiones con los objetivos de una asignatura, relacionados con el punto de vista del profesor. Un ejemplo de objetivo sería el siguiente: "presentar a los estudiantes los procedimientos de construcción básicos de túneles”. Como se puede comprobar, no es algo que se pueda evaluar. En cambio "elegir los procedimientos y la maquinaria más adecuada para la excavación de túneles” sí que se encuentra relacionado con lo que puede lograr el estudiante, siendo evaluable. También se podrían diferenciar los resultados de aprendizaje de un módulo, de una materia o de una asignatura. Además, cada resultado de aprendizaje de una asignatura se puede alcanzar con resultados de aprendizaje de segundo nivel correspondiente a las clases teóricas o las prácticas de aula, según se muestra en la Figura 1. Como se puede ver, los resultados del aprendizaje están directamente vinculados con una estrategia concreta de enseñanza y con unos métodos específicos de evaluación (Pellicer et al., 2016). Este alineamiento entre resultados, actividades de enseñanza y estrategias de evaluación dota de transparencia el proceso global de enseñanza- aprendizaje y permite garantizar la coherencia interna de los módulos y las asignaturas.

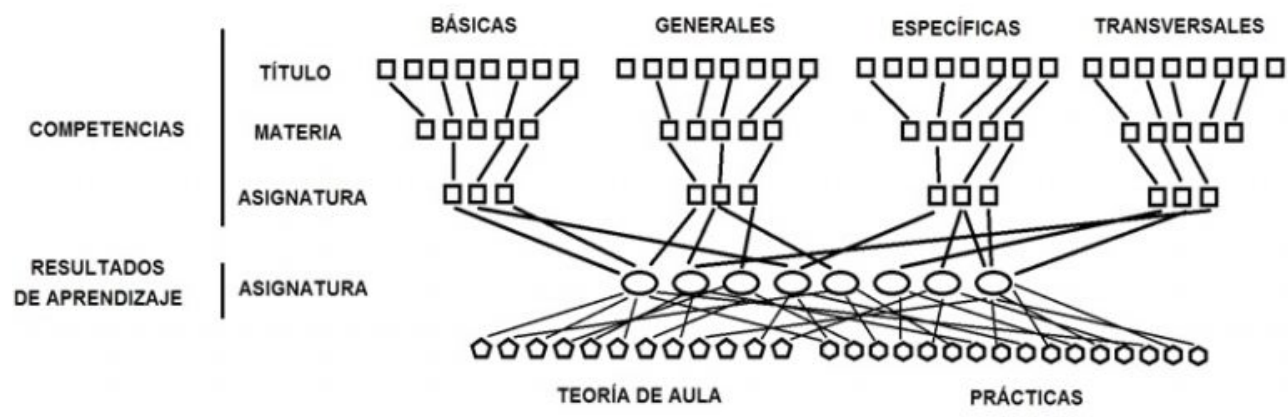

Fig. 1 Estructura de competencias y resultados de aprendizaje

Por otra parte, el contexto difumina en ocasiones la frontera entre resultado de aprendizaje y competencia. De hecho, hay países donde ambos conceptos son sinónimos. Así, el Marco Europeo de Cualificaciones para el aprendizaje permanente (EQF) considera que las competencias son parte de los resultados del aprendizaje. En cambio, el proyecto Tuning (Estructuras educativas en Europa) considera que las competencias se dividen en específicas y genéricas e incluyen “conocimientos y comprensión”, "saber cómo actuar” y

(cc) EY-NC-ND 2018, Universitat Politècnica de València

Congreso IN-RED (2018) 
"saber cómo ser", mientras que los resultados del aprendizaje, por su parte, expresan el nivel de competencia adquiridos. Sin embargo, en el Marco de Cualificaciones del EEES los resultados del aprendizaje son el producto del proceso de enseñanza, mientras que el término competencias se utiliza en un sentido amplio permitiendo la graduación de habilidades o destrezas, y se considera que está incluido en el concepto de resultados del aprendizaje.

En España, el uso del término "competencias" está más extendido que el de "resultados del aprendizaje”. Así, el R.D. 1393/2007 señala que "los planes de estudios conducentes a la obtención de un título deberán tener en el centro de sus objetivos la adquisición de competencias por parte de los estudiantes, ampliando, sin excluir, el tradicional enfoque basado en contenidos y horas lectivas". Los resultados del aprendizaje se mencionan en la exposición de motivos ("Se proponen los créditos europeos, ECTS, como unidad de medida que refleja los resultados del aprendizaje y volumen de trabajo realizado por el estudiante para alcanzar los objetivos establecidos en el plan de estudios") y en el punto 8 del Anexo I (la universidad debe presentar el "procedimiento general de la Universidad para valorar el progreso y los resultados del aprendizaje de los estudiantes"). Las órdenes ministeriales relacionadas con los títulos que habilitan para una profesión regulada se refieren a competencias, y no a resultados de aprendizaje. Éste último término sí que se utiliza en el R.D. 1027/2011 por el que se establece el Marco Español de Cualificaciones para la Educación Superior (MECES).

Ante esta confusión, se propone seguir el criterio de ANECA (2014), en donde se considera que los resultados del aprendizaje son concreciones de las competencias para un determinado nivel y que son el resultado del proceso de enseñanza-aprendizaje. Por tanto, se considera que los resultados del aprendizaje describen lo que los estudiantes deben ser capaces de hacer al término del proceso formativo o de la asignatura. Para que ello sea posible deben definirse con claridad; deben ser observables y evaluables; deben ser factibles y alcanzables por los estudiantes; deben diseñarse para asegurar su idoneidad y relevancia con respecto a la asignatura o enseñanza; deben guardar relación directa con los resultados del aprendizaje del programa; y deben corresponder al nivel definido en el MECES.

Los resultados del aprendizaje se definen con frases con un verbo de acción, un contenido u objeto sobre el que el estudiante tiene que actuar y un contexto o condiciones en las que se producirá la acción. Suele utilizarse la jerarquía de Bloom para su redacción. Remitimos al lector a la "Guía de apoyo para la redacción, puesta en práctica y evaluación de los resultados del aprendizaje” (ANECA, 2014).

Otro aspecto trascendental es la relación entre los resultados de aprendizaje y su evaluación (Yepes, 2017). En este sentido, los métodos y las actividades formativas y los sistemas de evaluación deben coordinarse para alcanzar los resultados del aprendizaje. Así, la ANECA considera importante esta alineación, como así queda reflejado en el "Criterio 6. Resultados de aprendizaje", en donde la directriz 6.1 a valorar para su cumplimiento indica "Las actividades formativas, sus metodologías docentes y los sistemas de evaluación empleados son adecuados y se ajustan razonablemente al objetivo de la adquisición de los resultados 
de aprendizaje previstos.” Por último, señalar que los resultados de aprendizaje son dinámicos, siendo aconsejable su revisión periódica y sistemática así como su interrelación con las metodologías docentes y las prácticas de evaluación.

\section{Resultados}

Lo anteriormente expuesto permite un proceso de reflexión que concluye en la formulación de los resultados de aprendizaje de primer y segundo nivel y su relación jerárquica con las competencias de distinto tipo establecidas en una asignatura, materia o título. A continuación se recoge como resultado de esta formulación su aplicación a las asignaturas de "Procedimientos de Construcción I y II" del Grado de Ingeniería Civil de la UPV. Los programas de estas asignaturas, que constituyen la base de esta formulación, se desarrollaron basándose en los contenidos determinados en la Memoria de Verificación del título oficial de "Graduado o Graduada en Ingeniería Civil por la Universitat Politècnica de València" (2502507) que fue aprobado por el Consejo de Universidades el 5 de noviembre de 2010, y cuya renovación de la acreditación del título oficial se resolvió favorablemente el 22 de julio de 2016. Se trata de asignaturas de la materia "Bases de la ingeniería de la construcción” perteneciente a la titulación del GIC, y por tanto es obligatoria para todos los estudiantes de esta titulación y se imparten en el segundo curso.

\subsection{Competencias de la materia "Bases de la Ingeniería de la Construcción"}

Las asignaturas "Procedimientos de Construcción I y II" pertenecen a la materia de carácter obligatorio "Bases de la Ingeniería de la Construcción". Cada una de ellas tiene una asignación de 4,5 créditos ECTS, repartidos en 2,5 créditos de teoría y 2,0 de prácticas. Además, también pertenecen a esta materia las siguientes asignaturas: "Electrotecnia", con 4,5 ECTS; "Materiales de construcción y sus aplicaciones a la ingeniería civil", con 6,0 ECTS; y "Química para la ingeniería civil", con 6,0 ECTS. Las competencias asignadas a esta materia, de acuerdo con la Memoria de Verificación, son:

\section{Competencias básicas y generales:}

A01 - Analizar críticamente los procesos propios de la Ingeniería Civil.

A02. - Aprender de manera autónoma nuevos conocimientos y técnicas adecuados para la Ingeniería Civil.

A03 - Comprender y asumir la responsabilidad ética y profesional de la actividad del Ingeniero Civil.

A04 - Comprender y utilizar el lenguaje propio de la ingeniería así como la terminología propia de la Ingeniería Civil.

A07 - Conocer y comprender las ciencias y las tecnologías correspondientes para la planificación, proyecto, construcción y explotación de las obras propias del Sector de la Ingeniería Civil. 
A08 - Dirigir y coordinar grupos de trabajo en el ámbito de la Ingeniería Civil, proponiendo métodos de trabajo estándar y herramientas a utilizar.

A10 - Tener la capacidad para organizar y gestionar técnica, económica y administrativamente los distintos medios de producción propios de la Ingeniería Civil.

A11 - Capacitar científica y técnicamente para el ejercicio de la profesión de Ingeniero Técnico de Obras Públicas con conocimiento de las funciones de asesoría, análisis, diseño, cálculo, proyecto, construcción, mantenimiento conservación y explotación.

A12 - Comprender los múltiples condicionamientos de carácter técnico y legal que se plantean en la construcción de una obra pública, y capacitación para emplear métodos contrastados y tecnologías acreditadas, con la finalidad de conseguir la mayor eficacia de la construcción dentro del respeto por el medio ambiente y la protección de la seguridad y salud de los trabajadores y usuarios de la obra pública.

\section{Competencias específicas:}

C02 - Comprender las propiedades químicas, físicas, mecánicas y tecnológicas de los materiales más utilizados en construcción.

C03 - Aplicar los conocimientos de materiales de construcción en sistemas estructurales, a partir del conocimiento de la relación entre la estructura de los materiales y las propiedades mecánicas que de ella se derivan.

C09 - Analizar la problemática de la seguridad y salud en las obras de construcción.

C10 - Comprender el sistema eléctrico de potencia: generación de energía, red de transporte, reparto y distribución, así como sobre tipos de líneas y conductores. Comprender la normativa sobre baja y alta tensión.

C12 - Comprender los procedimientos constructivos, la maquinaría de construcción y las técnicas de organización, medición y valoración de obras.

Estas competencias se desarrollan en las distintas asignaturas de la materia, según se muestra en la Tabla 1, según consta en la Memoria de Verificación del título y en las Guías Docentes de las asignaturas.

Tabla 1. Asignación de competencias en la materia "Bases de la ingeniería de la construcción” del Grado de Ingeniería Civil

\begin{tabular}{|c|c|c|c|c|c|c|c|c|c|c|c|c|c|c|}
\hline & \multicolumn{14}{|c|}{ Competencias } \\
\hline & \multicolumn{9}{|c|}{ Básicas y generales } & \multicolumn{5}{|c|}{ Específicas } \\
\hline Asignatura & A01 & A02 & A03 & A04 & A07 & A08 & A10 & A11 & A12 & $\mathrm{C02}$ & $\mathrm{CO3}$ & C09 & C10 & C12 \\
\hline Electrotecnia & & & & $\bullet$ & & & & & & & & & $\bullet$ & \\
\hline $\begin{array}{l}\text { Materiales de } \\
\text { construcción y sus } \\
\text { aplicaciones a la I.C. }\end{array}$ & $\bullet$ & $\bullet$ & $\bullet$ & $\bullet$ & & & & $\bullet$ & $\bullet$ & $\bullet$ & $\bullet$ & & & \\
\hline $\begin{array}{l}\text { Procedimientos de } \\
\text { Construcción I }\end{array}$ & • & & & $\bullet$ & & & $\bullet$ & & $\bullet$ & & & $\bullet$ & & $\bullet$ \\
\hline
\end{tabular}




\begin{tabular}{|l|c|c|c|c|c|c|c|c|c|c|c|c|c|c|}
\hline $\begin{array}{l}\text { Procedimientos de } \\
\text { Construcción II }\end{array}$ & $\bullet$ & $\bullet$ & & $\bullet$ & $\bullet$ & $\bullet$ & $\bullet$ & $\bullet$ & & & & $\bullet$ & & $\bullet$ \\
\hline $\begin{array}{l}\text { Química para la } \\
\text { ingeniería civil }\end{array}$ & $\bullet$ & $\bullet$ & $\bullet$ & $\bullet$ & & & & & & $\bullet$ & $\bullet$ & & & \\
\hline
\end{tabular}

\subsection{Las competencias transversales en las asignaturas "Procedimientos de Construcción I y II"}

La ETSICCP ha escogido la asignatura “Procedimientos de Construcción II" como punto de control de la competencia CT09. Pero, además, se trabajan las competencias CT01 y CT06. Estas tres competencias también se trabajan en "Procedimientos de Construcción I". De acuerdo con esta asignación, la asignatura trabaja las competencias según lo acordado por la UPV (http://www.upv.es/contenidos/COMPTRAN/):

\section{CT01.- Comprensión e integración}

"Comprender quiere decir "percibir y tener una idea clara de lo que se dice, se hace o sucede o descubrir el sentido profundo de algo". Para demostrar que algo se ha comprendido, la persona identifica y recupera la información y la explica con sus propias palabras, interpretando e integrando las ideas desde su propia perspectiva”.

\section{CT06.- Trabajo en equipo y liderazgo}

"El trabajo en equipo implica crear y desarrollar un clima de confianza mutua entre los componentes que permita trabajar de forma responsable y cooperativa. El término más apropiado para describir esta situación es COMPARTIR: compartir conocimientos, compromiso y responsabilidad. Supone el reparto de tareas y roles y el respeto a las normas y reglas de juego establecidas por y para el grupo".

\section{CT09.- Pensamiento crítico}

“El pensamiento crítico va más allá de las destrezas del análisis lógico, ya que, implica poner en cuestión los supuestos subyacentes en nuestras formas habituales de pensar y actuar $y$, en base a ese cuestionamiento crítico, estar preparado para pensar y hace diferente. El pensamiento crítico es el pensamiento de los interrogantes: ¿por qué las cosas son así?, ¿por qué las cosas no pueden ser de otro modo?, ¿por qué tú crees que son así?, etc. En consecuencia, diremos que una persona lo ha desarrollado en la medida en que se interroga sobre las cosas y se interesa por los fundamentos en los que se asientan las ideas, las acciones, las valoraciones y juicios tanto propios como ajenos."

Puesto que la asignatura "Procedimientos de Construcción II" se imparte en $2^{\circ}$ del GIC, se ha de evaluar el Nivel 1 de dominio de la competencia transversal.

En el caso de la CT01, el estudiante ha de "describir, relacionar e interpretar situaciones y planteamientos sencillos”. Los indicadores son:

- Identificar correctamente las ideas o conceptos de un texto, problema, ejercicio...

- Explicar correctamente, con sus propias palabras, las ideas planteadas en un texto, problema, ejercicio...

(cc) EY-NC-ND 2018, Universitat Politècnica de València 
- Interpretar, expresando de modo personal, las ideas o conceptos planteados en un texto, problema, ejercicio...

- Integrar distintas ideas o conceptos de la asignatura en sus análisis.

En el caso de la CT06, el estudiante ha de "participar y colaborar activamente en las tareas del equipo, orientándose hacia el trabajo en común". Los indicadores son:

- Aceptar y cumplir los objetivos del equipo.

- Acudir y participar activamente en las reuniones del equipo.

- Realizar las tareas asignadas dentro del equipo en el plazo fijado.

En el caso de la CT09, el estudiante ha de "mostrar una actitud crítica ante la realidad, siendo capaz de analizar y cuestionar información, resultados, conclusiones y otros puntos de vista”. Los indicadores son:

- Mostrar una actitud crítica ante la realidad: se pregunta el porqué de las cosas.

- Profundizar en un tema con lógica e imparcialidad, contrastando información en fuentes fiables.

- Diferenciar hechos de opiniones, interpretaciones o valoraciones.

- Prever las consecuencias (implicaciones prácticas) de las decisiones.

En la metodología docente se proponen las actividades desarrolladas relacionadas con la adquisición de cada competencia y los criterios de evaluación. El candidato, junto con otros profesores de la asignatura, analizaron en distintos trabajos la valoración del pensamiento crítico como competencia transversal basada en la percepción de los estudiantes respecto a su importancia, tanto en los grados de Ingenieros de Obras Públicas como los de Ingeniería Civil (Halpern, 1989; Martí y Yepes, 2015; Yepes y Martí, 2015; Yepes et al., 2016; Yepes et al., 2017). Los resultados muestran como casi tres cuartas partes de los estudiantes han alcanzado suficientemente la competencia. Sin embargo, los resultados muestran cierta desconexión entre los resultados relativos a las competencias específicas y los resultados relativos a la competencia transversal. Estas evidencias manifiestan que la adquisición de la competencia transversal del pensamiento crítico se ve favorecida por los trabajos de discusión en grupo. No obstante, la adquisición de competencias específicas por parte de los estudiantes requiere no sólo de trabajos en grupo, sino también de trabajos individuales.

\subsection{Propuesta de resultados de aprendizaje de la asignatura "Procedimientos de Construcción I"}

Los resultados de aprendizaje propuestos para "Procedimientos de Construcción I" considera las competencias y los contenidos de la asignatura, influyendo los criterios de evaluación seguidos para su consecución. En la Tabla 2 se recogen las relaciones entre los resultados de aprendizaje y las competencias asociadas. A modo de resumen, al terminar con éxito esta asignatura los estudiantes serán capaces de "comprender los diferentes 
procedimientos constructivos y aplicar la maquinaria y los medios auxiliares necesarios para realizar dichos trabajos, especialmente en lo relativo a los sondeos y mejora de terrenos, las excavaciones y voladuras y a la ejecución de firmes, así como entender el funcionamiento de las instalaciones de fabricación de áridos y de aglomerado asfáltico".

Tabla 2. Resultados de aprendizaje de la asignatura "Procedimientos de Construcción I"

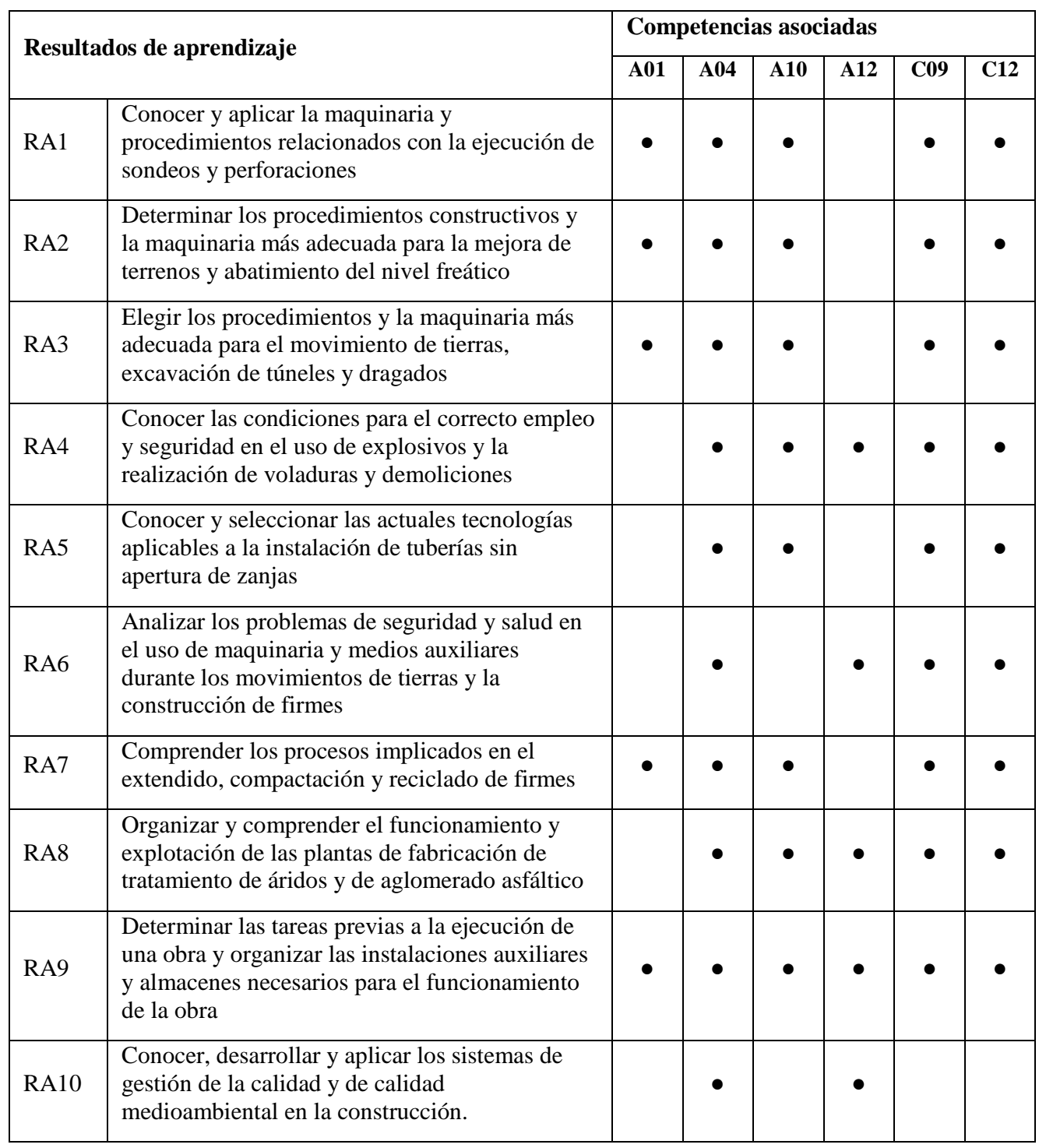

\subsection{Propuesta de resultados de aprendizaje de la asignatura "Procedimientos de Construcción II"}

Los resultados de aprendizaje propuestos para "Procedimientos de Construcción II", análogamente al caso anterior, se han formulado considerando las competencias y los contenidos de la asignatura, influyendo asimismo los criterios de evaluación. La Tabla 3 recoge las relaciones entre los resultados de aprendizaje y las competencias asociadas. De

(cc) EY-NC-ND 2018, Universitat Politècnica de València 
este modo, al terminar esta asignatura los estudiantes serán capaces de "comprender los diferentes procedimientos constructivos y aplicar la maquinaria y los medios auxiliares necesarios para realizar dichos trabajos, especialmente en lo relativo a la ejecución de cimentaciones y estructuras, así como entender el funcionamiento de las instalaciones de fabricación de hormigón y de prefabricados, evaluar los costes y la producción de los equipos y organizar y planificar las obras".

Tabla 3. Resultados de aprendizaje de la asignatura "Procedimientos de Construcción II"

\begin{tabular}{|c|c|c|c|c|c|c|c|c|c|c|}
\hline \multirow{2}{*}{\multicolumn{2}{|c|}{ Resultados de aprendizaje }} & \multicolumn{9}{|c|}{ Competencias asociadas } \\
\hline & & \multirow{2}{*}{$\begin{array}{c}\text { A } \\
01\end{array}$} & \multirow{2}{*}{$\begin{array}{l}\mathbf{A} \\
\mathbf{0 2} \\
\\
\end{array}$} & \multirow{2}{*}{$\begin{array}{l}\text { A } \\
04 \\
\\
\end{array}$} & \multirow{2}{*}{$\begin{array}{c}\mathbf{A} \\
\mathbf{0 7}\end{array}$} & \multirow{2}{*}{$\begin{array}{c}\text { A } \\
\mathbf{0 8}\end{array}$} & \multirow{2}{*}{$\begin{array}{c}\text { A } \\
10 \\
\\
\end{array}$} & \multirow{2}{*}{$\begin{array}{l}\text { A } \\
11 \\
\bullet\end{array}$} & \multirow{2}{*}{$\begin{array}{c}\mathrm{C} \\
09\end{array}$} & \multirow{2}{*}{$\begin{array}{r}\mathrm{C} \\
12 \\
\\
\end{array}$} \\
\hline RA1 & $\begin{array}{l}\text { Evaluar los rendimientos, la } \\
\text { productividad y los costes de los } \\
\text { equipos empleados en obra }\end{array}$ & & & & & & & & & \\
\hline RA2 & $\begin{array}{l}\text { Conocer el mantenimiento y la } \\
\text { explotación de la maquinaria en } \\
\text { función de sus características y del } \\
\text { uso al que vayan a estar sometidos }\end{array}$ & & & $\bullet$ & • & & $\bullet$ & $\bullet$ & & $\bullet$ \\
\hline RA3 & $\begin{array}{l}\text { Identificar los tipos y } \\
\text { características de la maquinaria y } \\
\text { de los medios auxiliares, su } \\
\text { funcionamiento y aplicabilidad en } \\
\text { las obras }\end{array}$ & & & $\bullet$ & & & $\bullet$ & $\bullet$ & & $\bullet$ \\
\hline RA4 & $\begin{array}{l}\text { Comprender y aplicar los } \\
\text { conceptos relacionados con la } \\
\text { teoría y práctica de los cables y la } \\
\text { maquinaria de elevación }\end{array}$ & & & $\bullet$ & & & $\bullet$ & $\bullet$ & & $\bullet$ \\
\hline RA5 & $\begin{array}{l}\text { Organizar y comprender el } \\
\text { funcionamiento y explotación de } \\
\text { las plantas de fabricación de } \\
\text { hormigón y de prefabricados }\end{array}$ & $\bullet$ & & $\bullet$ & $\bullet$ & $\bullet$ & $\bullet$ & $\bullet$ & & $\bullet$ \\
\hline RA6 & $\begin{array}{l}\text { Comprender los procesos } \\
\text { implicados en la fabricación, } \\
\text { transporte y puesta en obra del } \\
\text { hormigón }\end{array}$ & $\bullet$ & $\bullet$ & $\bullet$ & • & $\bullet$ & & $\bullet$ & & $\bullet$ \\
\hline RA7 & $\begin{array}{l}\text { Elegir los procedimientos } \\
\text { constructivos y la maquinaria más } \\
\text { adecuada para la construcción de } \\
\text { cimentaciones y estructuras }\end{array}$ & $\bullet$ & $\bullet$ & $\bullet$ & $\bullet$ & $\bullet$ & $\bullet$ & $\bullet$ & $\bullet$ & $\bullet$ \\
\hline RA8 & $\begin{array}{l}\text { Analizar los problemas de } \\
\text { seguridad y salud en el uso de } \\
\text { maquinaria y medios auxiliares al } \\
\text { ejecutar estructuras de hormigón }\end{array}$ & & $\bullet$ & • & & & & $\bullet$ & • & $\bullet$ \\
\hline RA9 & $\begin{array}{l}\text { Seleccionar, organizar y controlar } \\
\text { los recursos humanos y materiales } \\
\text { necesarios en la ejecución de una } \\
\text { unidad de obra }\end{array}$ & & $\bullet$ & 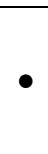 & $\bullet$ & $\bullet$ & $\bullet$ & $\bullet$ & $\bullet$ & $\bullet$ \\
\hline RA10 & $\begin{array}{l}\text { Aplicar las técnicas de } \\
\text { planificación y programación }\end{array}$ & $\bullet$ & $\bullet$ & $\bullet$ & $\bullet$ & & $\bullet$ & $\bullet$ & & $\bullet$ \\
\hline
\end{tabular}




\begin{tabular}{|l|l|l|l|l|l|l|l|l|l|l|}
\hline $\begin{array}{l}\text { temporal de una obra a partir de } \\
\text { una documentación básica }\end{array}$ & & & & & & & & & \\
\hline
\end{tabular}

\subsection{Ejemplo de integración jerárquica de resultados de aprendizaje y competencias}

A continuación se presenta como ejemplo de integración jerárquica de resultados de aprendizaje de segundo nivel (procedentes de las teoría de aula y de las prácticas de aula) en un resultado de aprendizaje de primer nivel y cómo éste se relaciona con algunas de las competencias del grado. Se ha elegido para ello el resultado de aprendizaje RA4 de la asignatura "Procedimientos de Construcción I", que a su vez se corresponde con la material “Bases de la Ingeniería de la Construcción” del Grado de Ingeniería Civil. Este resultado de aprendizaje se puede formular de la siguiente forma: “conocer las condiciones para el uso correcto del empleo y seguridad en el uso de explosivos y la realización de voladuras y demoliciones". La adquisición de este resultado de aprendizaje depende directamente de los temas teóricos T9 “Explosivos y accesorios de voladuras” y T10 "Voladuras de rocas, voladuras especiales y demoliciones”, además de la práctica de aula PA13 “Diseño de una voladura en banco en una cantera". Los resultados de aprendizaje de segundo nivel se detallan en la Tabla 4. La relación entre los resultados de primer y segundo nivel, y las competencias, se representan en la Figura 2. El conjunto completo de correspondencias jerárquicas para las dos asignaturas se puede consultar en el trabajo de Yepes (2017).

Tabla 4. Resultados de aprendizaje de segundo nivel correspondientes con el resultado RA4 de la asignatura "Procedimientos de Construcción II"

\begin{tabular}{|c|c|}
\hline \multicolumn{2}{|c|}{ Resultados de aprendizaje RA4 } \\
\hline Tema T09 & $\begin{array}{l}\text { Identificar y conocer los materiales explosivos y los accesorios utilizados en los } \\
\text { procesos de voladura. } \\
\text { Advertir la peligrosidad y medidas de precaución a tener en cuenta en cada tipo de } \\
\text { explosivo. } \\
\text { Conocer la aplicabilidad de los detonadores y su cálculo. }\end{array}$ \\
\hline Tema T10 & $\begin{array}{l}\text { Identificar el equipo especializado que se utiliza en los procesos de voladura. } \\
\text { Conocer las precauciones a tener en cuenta en durante todo el proceso de una voladura, } \\
\text { antes y después de la pega. } \\
\text { Diseñar voladuras para la explotación de canteras, apertura de tajos y canales y } \\
\text { perforación de túneles. } \\
\text { Conocer las técnicas de voladuras especiales, tanto bajo el agua como en atmósferas } \\
\text { peligrosas. } \\
\text { Comprender de la reglamentación existente en materia de explosivos, así como su uso y } \\
\text { manejo. }\end{array}$ \\
\hline $\begin{array}{l}\text { Práctica de } \\
\text { Aula } \\
\text { PA13 }\end{array}$ & $\begin{array}{l}\text { Seleccionar el tipo de explosivo y los accesorios necesarios en cada barreno } \\
\text { Diseñar la voladura en banco atendiendo a sus parámetros principales } \\
\text { Entender la importancia del diámetro del barreno en el diseño de la voladura } \\
\text { Calcular la perforación específica y la carga específica }\end{array}$ \\
\hline
\end{tabular}

(cc) EY-NC-ND 2018, Universitat Politècnica de València

Congreso IN-RED (2018) 
En la Tabla 2 se puede comprobar que el resultado de aprendizaje RA4 se encuentra relacionado con las competencias A04, A10, C09 y C12. Toda esta información se resume en la Figura 2, donde se grafían las relaciones entre los resultados de aprendizaje de primer nivel (RA4), de segundo nivel y las competencias correspondientes (Yepes, 2018).

El último paso sería establecer un sistema de evaluación que permitiese garantizar la adquisición del resultado de aprendizaje de primer nivel. Si observamos en la Tabla 4, la pormenorización en resultados de aprendizaje de segundo nivel permite, casi de forma directa, formular pruebas de evaluación.

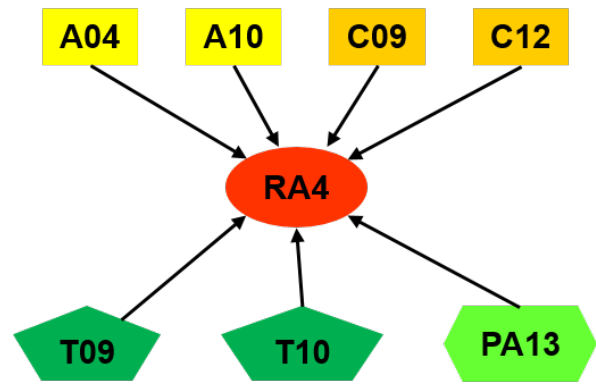

Fig. 2 Correspondencias jerárquicas entre un resultado de aprendizaje de primer nivel, las competencias y los resultados de aprendizaje de segundo nivel

\section{Conclusiones}

La comunicación se ha centrado, por una parte, en aclarar los conceptos de competencia y resultado de aprendizaje relacionados con una asignatura, muchas veces confusos en función del contexto. Para ello se ha optado por considerar que los resultados del aprendizaje son concreciones de las competencias para un determinado nivel y que son el resultado del proceso de enseñanza-aprendizaje. Por otra parte, se ha comprobado cómo las competencias se pueden clasificar y jerarquizar atendiendo al contexto del título, materia o asignatura. Lo mismo ocurriría con los resultados de aprendizaje que, en el caso de una asignatura, a su vez se pueden desglosar en resultados de segundo nivel en función de las clases teóricas o prácticas. Este desglose resulta necesario para poder diseñar sistemas de evaluación coherentes con la garantía en la adquisición de los resultados de aprendizaje, y por ende, de las competencias. Para que todo ello sea posible, es necesario establecer matrices de correspondencias jerárquicas entre las competencias y los resultados de aprendizaje. Como resultado de lo anterior, se ha presentado la aplicación práctica de esta propuesta en dos asignaturas, "Procedimientos de Construcción I y II" del Grado de Ingeniería Civil de la UPV. 


\section{Agradecimientos}

El autor agradece el apoyo recibido por la Universitat Politècnica de València (Equipo de Innovación y Calidad Educativa EXCELCON):

\section{Referencias}

ANECA (2014). Guía de apoyo para la redacción, puesta en práctica y evaluación de los resultados de aprendizaje. Madrid. Disponible en <http://www.aneca.es/Documentos-y-publicaciones/Otrasguias-y-documentos-de-evaluacion/Guia-de-apoyo-para-la-redaccion-puesta-en-practica-yevaluacion-de-los-RESULTADOS-DEL-APRENDIZAJE> [Consulta: 5 de marzo de 2018]

España. Orden CIN/307/2009, de 9 de febrero, por la que se establecen los requisitos para la verificación de los títulos universitarios oficiales que habiliten para el ejercicio de la profesión de Ingeniero Técnico de Obras Públicas. BOE, 18 de febrero de 2009, núm. 42, p. 17166-17170.

España. Real Decreto 1027/2011, de 15 de julio, por el que se establece el Marco Español de Cualificaciones para la Educación Superior. BOE, 3 de agosto de 2011, núm. 185, p. 87912-87918.

España. Real Decreto 1393/1997, de 29 de octubre, por el que se establece la ordenación de las enseñanzas universitarias oficiales. BOE, 30 de octubre de 2007, núm. 260, p. 44037-44048.

HALPERN, D.F. (1989). Thought and Knowledge: An Introduction to Critical Thinking. Hillsdale, New Jork: Erlbaum.

LASNIER, F. (2000). Réussir la formation par compétences. Montréal: Guérin.

MARTÍ, J.V. y YEPES, V. (2015). "Pensamiento crítico como competencia transversal en el grado de Ingeniería de Obras Públicas: valoración previa” en Congreso In-Red 2015. Valencia, pp. 1-12. Disponible en: <http://dx.doi.org/10.4995/INRED2015.2015.1560> [Consulta: 7 de marzo de 2018]

MINISTERIO DE EDUCACIÓN, CULTURA Y DEPORTE (2003). La integración del sistema universitario español en el Espacio Europeo de Enseñanza Superior. Documento-Marco. Disponible en <http://www.eees.es/pdf/Documento-Marco_10_Febrero.pdf> [Consulta: 6 de marzo de 2018]

PELLICER, E., SIERRA, L.A. y YEPES, V. (2016). “Appraisal of infrastructure sustainability by graduate students using an active-learning method" en Journal of Cleaner Production, vol. 113, p. 884-896.

PELLICER, E., YEPES, V. y ORTEGA, A.J. (2013). “Method for planning a graduate program in construction management” en Journal of Professional Issues in Engineering Education and Practice ASCE, vol. 139, issue 1, p. 33-41.

TORRES-MACHÍ, C., CARRIÓN, A., YEPES, V. y PELLICER, E. (2013). "Employability of graduate students in construction management” en Journal of Professional Issues in Engineering Education and Practice ASCE, vol. 139, issue 2, p. 163-170.

UNIVERSITAT POLITÈCNICA DE VALÈNCIA (2006). Guía docente de la UPV: criterios para su elaboración. Plan de acciones para la convergencia europea (PACE). Valencia: Instituto de Ciencias de la Educación. Disponible en: http://www.aqu.cat/doc/doc_52850666_1.pdf [Consulta: 6 de marzo de 2018]

UNIVERSITAT POLITÈCNICA DE VALÈNCIA. Competencias transversales. Disponible en: $<$ http://www.upv.es/contenidos/COMPTRAN/> [Consulta: 6 de marzo de 2018]

(cc) EY-NC-ND 2018, Universitat Politècnica de València

Congreso IN-RED (2018) 
VILA, A. y POBLETE, M. (2011). "Evaluación de competencias genéricas: principios, oportunidades y limitaciones” en Bordón, vol. 63, issue 1, p. 147-170.

YEPES, V. (2017). Proyecto docente. Concurso de acceso al cuerpo de catedráticos de universidad. Valencia: Universitat Politècnica de València.

YEPES, V. (2018) "Los resultados de aprendizaje de la asignatura «Procedimientos de Construcción»” en El Blog de Víctor Yepes, 16 de enero $<$ http://victoryepes.blogs.upv.es/2018/01/16/los-resultados-de-aprendizaje-de-la-asignaturaprocedimientos-de-construccion/> [Consulta: 5 de marzo 2018]

YEPES, V., PELLICER, E. y ORTEGA, J.A. (2012). "Designing a benchmark indicator for managerial competences in construction at the graduate level” en Journal of Professional Issues in Engineering Education and Practice ASCE, vol. 138, issue 2, p. 48-54.

YEPES, V. y MARTÍ, J.V. (2015). “Competencia transversal ‘pensamiento crítico’ en el grado de ingeniería civil: valoración previa” en XIII Jornadas de Redes de Investigación en Docencia Universitaria. Alicante, pp. 2944-2952. Disponible en <http://rua.ua.es/dspace/handle/10045/49970> [Consulta: 7 de marzo de 2018]

YEPES, V., SEGADO, S., PELLICER, E. y TORRES-MACHÍ, C. (2016). "Acquisition of competences in a Master Degree in Construction Management" en 10th International Technology, Education and Development Conference (INTED 2016). Valencia, pp. 718-727. Disponible en <https://library.iated.org/view/YEPES2016ACQ> [Consulta: 6 de marzo de 2018]

YEPES, V., MARTÍ, J.V. y MOLINA-MORENO, F. (2017). “Transverse competence 'critical thinking' in civil engineering graduate studies: preliminary assessment” en 11th annual International Technology, Education and Development Conference (INTED 2017). Valencia, pp. 2639-2649. Disponible en <https://library.iated.org/view/YEPES2017TRA> [Consulta: 7 de marzo de 2018] 